\title{
Measuring self-reported ability to perform activities of daily living: a Rasch analysis
}

\author{
Eva Ejlersen Wæhrens ${ }^{1,2^{*}} \mathbb{D}$, Anders Kottorp ${ }^{3}$ and Kristina Tomra Nielsen ${ }^{1,4}$
}

\begin{abstract}
Background: Since the number of persons diagnosed with multi-morbidity is increasing, there is a need for generic instruments to be able to assess, measure and compare ADL ability across diagnoses. Accordingly, the ADL-Interview (ADL-I) was developed to be used in rehabilitation research and clinical practice. The aim of this study was to investigate if the ADL-I can be used to provide valid and reliable ADL ability measures across gender and diagnostic groups.

Methods: ADL-I data were extracted from an existing research database on persons with chronic conditions including medical, rheumatological, oncological, neurological, geriatric and psychiatric diagnoses. Data were analysed based on Rasch Measurement methods to examine: the psychometric properties of the rating scale; ADL item and person fit to the Rasch model; if the difficulty of the ADL tasks differs across gender and diagnostic groups, and if the ADL-I provides precise and reliable measures of ADL ability.

Results: Data on $n=2098$ persons were included in the final analysis. Initial evaluation of the $0-3$ rating scale revealed threshold disordering between categories 1 and 2. After removal of 16 underfitting items, the variance explained by the Rasch dimension increased from 54.3 to $58.0 \%$, thresholds were ordered, but the proportion of persons with misfitting ADL-I measures increased slightly from 8.7 to $9.1 \%$. The person separation index improved slightly from 2.75 to 2.99 (reliability $=0.90$ ). Differential test function analysis, however, supported that the 16 underfitting items did not represent a threat to the measurement system. Similarly, ADL items displaying differential item functioning across gender and diagnoses did not represent a threat to the measurement system. The ADL items and participants were well distributed along the scale, with item and person measures well targeted to each other, indicating a small ceiling effect and no floor effect.
\end{abstract}

Conclusions: The study results overall suggest that the ADL-I is producing valid and reliable measures across gender and diagnostic groups among persons within a broad range of ADL ability, providing evidence to support generic use of the ADL-I.

Trial registration: N/A.

\section{Background}

All human beings have a need to perform activities of daily living (ADL). A need perceived by the person and/ or by society. ADL includes both Personal ADL (PADL)

\footnotetext{
${ }^{*}$ Correspondence: eva.elisabet.waehrens@regionh.dk

${ }^{1}$ The ADL Unit, The Parker Institute, Copenhagen University Hospital Bispebjerg-Frederiksberg, Nordre Fasanvej 57, 2000 Frederiksberg, Denmark

Full list of author information is available at the end of the article
}

and Instrumental ADL (IADL). PADL tasks are typically performed on a daily basis, regardless of gender, housing conditions, culture and interests. PADL cover tasks such as toileting, bathing, dressing, and eating. IADL involves tasks that are necessary to live an independent life and cover more complex tasks including cleaning, shopping, and cooking [1]. The ability to perform ADL tasks are often affected by acute, long-term or chronic conditions. Thus, problems related to ADL are typically targeted in the rehabilitation process [2]. To plan and implement original author(s) and the source, provide a link to the Creative Commons licence, and indicate if changes were made. The images or other third party material in this article are included in the article's Creative Commons licence, unless indicated otherwise in a credit line to the material. If material is not included in the article's Creative Commons licence and your intended use is not permitted by statutory regulation or exceeds the permitted use, you will need to obtain permission directly from the copyright holder. To view a copy of this licence, visit http://creativecommons.org/licenses/by/4.0/. The Creative Commons Public Domain Dedication waiver (http://creativeco mmons.org/publicdomain/zero/1.0/) applies to the data made available in this article, unless otherwise stated in a credit line to the data. 
rehabilitation interventions it is therefore necessary to evaluate the clients' initial level of ADL ability.

Within the field of rehabilitation, several instruments used to evaluate ADL ability have been developed. Some are diagnosis-specific, others are generic i.e., for use across gender and diagnostic groups. The Barthel Index [3] and the Functional Independence Measure (FIM ${ }^{\mathrm{TM}}$ ) [4] are examples of generic instruments that are wellknown and commonly used within rehabilitation. In both instruments the ability to perform PADL tasks independently is evaluated based on an ordinal rating scale and the interpretation is based on summed scores. ADL ability, however, also involves performance of the more complex IADL tasks and need of help is only one of several aspects related to performance. Hence, decreased quality of ADL task performance may also be indicated by increased effort, inefficient use of time and safety risk.

The Assessment of Motor and Process Skills (AMPS) $[5,6]$ is an example of a generic observation-based instrument covering both PADL and IADL. Further, the instrument is developed to measure the quality of ADL task performance based on several aspects including physical effort, efficiency, safety and independence. The AMPS is developed based on Rasch measurement methods and when using the instrument, linear measures of both ADL motor and ADL process ability are generated based on an interval scale. The psychometric properties of the observation-based AMPS ADL ability measures have been established across gender and diagnostic groups [7]. Still, observation-based measures represent the outsider's perspective which has limited relationship to the insider's perspective i.e., the person's perceived ability [8-10]. Until recently, an ADL instrument, based on self-report, focused on the quality of performance and providing linear measures, was not available. Consequently, the ADLInterview (ADL-I) was developed $[8,11]$.

The ADL-I is an instrument developed to describe and measure the quality of ADL task performance based on self-report. When administering the ADL-I, persons are asked to evaluate the quality of their performance in 47 specific ADL tasks; 31 tasks related to PADL and 16 tasks related to IADL. The aspects of quality of performance evaluated in the ADL-I are similar to the aspects evaluated in the AMPS: physical effort, efficiency, safety and independence. ADL-I data can be used to generate an overall linear measure of self-reported quality of ADL task performance. Similar to the AMPS, Rasch measurement methods have been employed to develop the ADL-I [11]. So far, the ADL-I has been applied in research studies among various diagnostic groups e.g. rheumatic diseases [8], depression [9], chronic obstructive pulmonary disease (COPD) [12], advanced cancer $[10,13]$, a mixed sample of chronic conditions [14] and in a geriatric population [15]. For each of these research studies, study-specific ADL-I measures have been generated based on Rasch measurement methods, verifying validity and reliability of the measures. Since the ADL-I was developed as a generic instrument to also be used in clinical rehabilitation settings, representing a need to measure ADL ability across a diverse group of patients including patients with multimorbidity, the next step is to investigate if the ADL-I can be used to provide valid and reliable ADL ability measures across gender and diagnostic groups. Based on previous research [16] we did expect to find some variation in difficulty of ADL tasks across gender and diagnostic groups, but not to the point that it would affect the measurement system.

\section{Methods \\ Aim}

The overall aim was to examine the psychometric properties of the ADL-I applied among males and females living with various chronic conditions. More specifically, the following research questions were addressed.

- Does a four-category rating scale used with the ADL tasks (items) of the ADL-I demonstrate sound psychometric properties?

- Do the ADL tasks (items) define a single unidimensional construct?

- Do the person responses demonstrate expected and valid response-pattern?

- Does the difficulty of the ADL tasks (items) differ across gender and diagnostic groups?

- Does the ADL-I provide precise and reliable measures of ADL ability?

\section{Design and setting}

The study was a descriptive register-based study. All data was obtained from an existing research database at the ADL unit of the Parker Institute, Copenhagen University Hospital, Bispebjerg og Frederiksberg. The database contains data on self-reported ADL task performance using the ADL-I collected in a range of research studies since 2007 by occupational therapists, trained in administering the ADL-I. The database contains no client identifiers besides diagnosis, gender and age. Hence, anonymized data from various client groups is included in the database, e.g., persons with rheumatologic diseases, cancer, COPD, schizophrenia, depression, mild stroke and persons with geriatric or orthopaedic problems.

\section{Participants/materials}

Self-reported ADL ability reflects the person's perceived ability and is based on experiences of performance. 
Persons with decreased ADL ability due to acute illness will have very limited experience with their present ability to perform ADL task. Accordingly, only ADL-I data on diagnostic groups considered to be living with chronic conditions were included. Chronic conditions were defined by Goodman et al. as "conditions that last a year or more and require ongoing medical attention and/or limit activities of daily living" [17]. Hence, data on persons with medical, rheumatological, oncological, neurological, geriatric and psychiatric chronic conditions were extracted from the abovementioned database. Subsequently, persons with maximum scores were excluded. In order to characterize the study sample, demographic data (gender and age) was also extracted from the database.

\section{Instrumentation}

The ADL-Interview (ADL-I) [18] is an occupational therapy evaluation tool developed to describe and measure the quality of ADL task performance based on selfreport (i.e. ADL ability). When administering the ADL-I, persons are asked to evaluate the quality of their performance in 47 ADL tasks; 31 tasks related to PADL and 16 tasks related to IADL. The ADL tasks are defined are organised into 12 ADL domains; Eating and drinking, Mobility, Going to the toilet, Dressing, Personal Hygiene, Grooming, Communication, Transportation, Cooking, Shopping, Cleaning and Washing, based on the ADL Taxonomy $[19,20]$.

When reporting the quality of ADL task performance, the person uses seven response categories: (a) I perform the task independently without the use of extra time or effort and without risk; (b) I perform the task independently without the use of extra time or effort and without risk, but I use helping aids; (c) I perform the task independently, but it takes me extra time; (d) I perform the task independently, but I use extra effort/get tired faster; (e) I perform the task independently, but there is a risk that I might hurt myself; (f) I need assistance from someone, but I do participate; and (g) The task is performed by others for me-I cannot participate actively. For clinical purposes, the persons can use more than one response category if several apply to their performance of the specific ADL task. If a person finds a task irrelevant to his or her daily life, the response category "Not relevant" is used.

Evaluation of the quality of PADL task performance is based on the past $24 \mathrm{~h}$, whereas quality of IADL task performance is based on the past week. ADL-I data can be used to describe task-specific self-reported quality of ADL task performance in a single person or a group of persons as well as to generate an overall measure of self-reported quality of ADL task performance. To generate overall linear measures, the mark given in the lowest response category on each task for each person is rated using a four-point ordinal rating scale: Competent $($ score $=3$ ) covering response categories (a) and (b), Using extra time/effort $($ score $=2)$ covering response categories (c) and (d), Need for help/safety (score $=1)$ covering response categories (e) and (f), and Unable (score $=0$ ) covering response category (g). The ordinal scores are then, based on the Rasch rating scale model, transformed into an overall linear (interval scale) measure of selfreported quality of ADL task performance, adjusted for the difficulty of the ADL tasks.

The ADL-I was initially developed and validated in persons with various types of rheumatic diseases [8]. Later studies have supported the validity of the ADL-I to describe and measure self-reported quality of ADL task performance in persons with other chronic conditions e.g. depression [9], COPD [12] and incurable cancer [10, 13]. Further, a study suggested that the ADL-I is sensitive to change in ADL ability among older persons participating in a reablement program [15].

\section{Data analyses}

The Rasch computer program, WINSTEPS version 4.7.0 [21] was used to convert ordinal scores into equal interval units or measures of the person's self-reported overall quality of ADL task performance. The conversions are based on log-odds probabilities; thus, the item difficulty and the quality of ADL task performance measures are expressed in logits (log-odds probability units) [22]. The measures of item difficulty, and quality of ADL task performance, respectively, represent item and person location along the linear scale. WINSTEPS was also used to generate several statistics to evaluate aspects of validity and reliability, including fit of the data to the Rasch model assertions [23, 24]. An overview of the analysis is provided in Table 1. Details related to Rasch analysis procedures have been described elsewhere [23-25].

Within Rasch measurement methods, the Partial Credit Model (PCM) [26] and the Rasch Rating Scale Model (RSM) [27] are applied with data derived from response scales with more than two categories. The only difference between the two models is related to their assumptions about distance between the response categories. The PCM assumes that the distance between the response categories is not the same, whereas the RSM assumes equal distances between categories. Evaluation of the log likelihood ratio indicated fit to an interval model $(p=0.3548)$, thus, the RSM was applied. The RSM for ADL-I includes two facets (items and persons) and is based on two assertions: (a) a person experiencing more quality of ADL task performance is more likely to receive higher ratings on harder ADL-I items than a person experiencing lower quality of ADL task performance; and (b) 


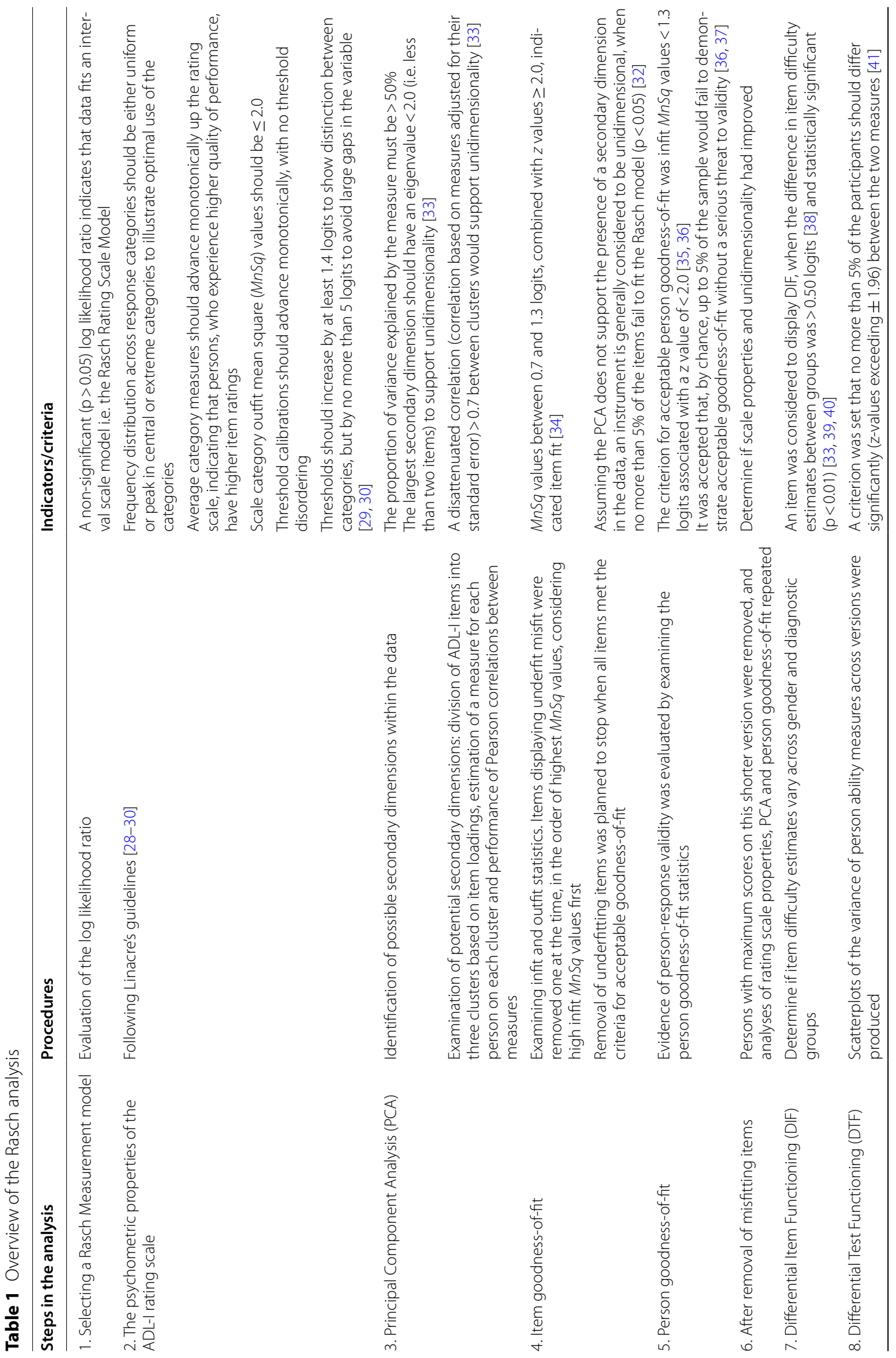




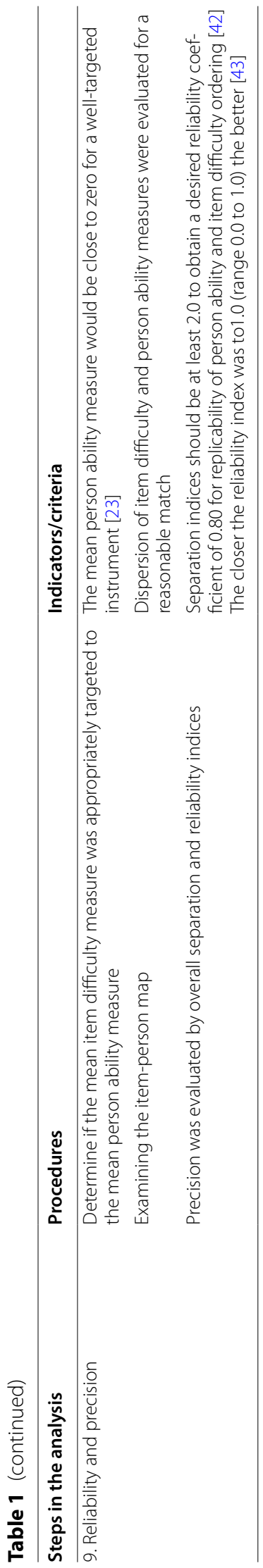


any person is more likely to receive higher ratings on easier ADL-I items than on harder ADL-I items [23]. When data meet these expectations, the items and the persons fit the measurement model, supporting internal scale and person response validity of the ADL-I, respectively.

Prior to the RSM analysis, persons with maximum scores on ADL-I were removed, since they mathematically correspond to infinite or indefinite measures on the latent variable and so are not directly estimable [25]. To address the first research question, Linacre's guidelines [28-30] for evaluation of the psychometric properties of a rating scale was applied. The second research question, related to determine if items in the ADL-I represent a single unidimensional construct, was addressed in several analyses including a Principal Component Analysis (PCA) of the standardized residuals (i.e. the difference between what the Rasch model predicts and what was observed), item and person goodness-of-fit and Differential Item Functioning (DIF) for gender and diagnostic groups [31, 32]. Moreover, since the clinical relevance of the tasks (items) included in the ADL-I has already been verified in previous studies $[19,20]$, it was investigated whether the measurement system would be disrupted if clinically relevant, but misfitting items and/or items displaying DIF were retained. This was done by evaluating for Differential Test Functioning (DTF) [31].

The PCA of the standardized residuals was performed to identify possible secondary dimensions within the data. The analyses of goodness-of-fit to the Rasch model included both infit and outfit statistics. While the infit statistics are more sensitive to unexpected patterns of observations on items that are roughly targeted to the people, outfit statistics are more sensitive to unexpected observations on items that are very easy or very hard [33]. Also, both underfit and overfit to the model was identified [23]. While underfit degrades the quality of measures, overfit in general has no practical implications, but might be an indication of lack of local independence (i.e. significant correlations among the items after the contribution of the underlying construct is removed). Therefore, items displaying overfit misfit were not considered a threat to the measurement system and retained in the instrument. Subsequently, the investigation of unidimensionality was continued by evaluation of personresponse validity and analyses of DIF. DIF occurs when item difficulty estimates vary between groups, thus representing a risk to the unidimensionality requirement. To determine if the ADL-I can be used as a generic tool to measure self-reported quality of ADL task performance, DIF was evaluated based on gender and diagnosis.

When items displaying misfit had been identified and removed, persons with maximum scores on this shorter version of ADL-I were removed, and analyses of the psychometric properties of the rating scale, PCA, person goodness-of-fit and DIF were repeated, to determine if scale properties and unidimensionality had improved.

Afterwards, analyses of DTF were performed to determine if relevant, but removed, items would disrupt the measurement system if kept in the instrument. DTF occurs when person ability measures vary between two versions of a test. The evaluation of DTF related to inclusion or omission of misfitting ADL items was performed by comparing (a) measures of quality of ADL task performance based on a version of ADL-I containing only items displaying fit to the Rasch rating scale model to (b) measures of quality of ADL task performance based on a version of ADL-I containing all 47 items. Similarly, it was investigated whether retaining the items displaying DIF for gender or diagnosis would disrupt the measurement system by means of DTF. By comparing (a) quality of ADL task performance measures based on gender- or diagnosis specific item calibrations with (b) quality of ADL task performance measures based on common item calibrations, the variance of measures across a gender- or diagnosis-specific version and a common version could be explored, for male, females and the six diagnostic subgroups.

Finally, the last research question concerned the precision and reliability of the ADL-I measures. First, it was evaluated whether the mean ADL item difficulty measure was appropriately targeted to the mean quality of ADL task performance measure of the participants. Second, the dispersion of the ADL item difficulty and quality of ADL task performance measures was evaluated for a reasonable match by examining the item-person map, a graphic display of the distribution of item and person measures, generated by the WINSTEPS program. Precision was evaluated by overall separation and reliability indices.

\section{Results}

Data on $n=2198$ persons were extracted from the database, based on the inclusion criteria. After removing $\mathrm{n}=60(2.7 \%)$ persons with maximum scores on the ADL-I, the study sample used in the initial analyses was $\mathrm{n}=2138$ persons representing six diagnostic groups (Table 2). Later, after removal of items displaying misfit, data on another $\mathrm{n}=40$ (1.9\%) persons with maximum scores on the ADL-I were removed from the final analyses $(n=2098)$. Thus, a total of $n=100$ persons, of which $\mathrm{n}=48$ were women, were removed from the analyses representing all diagnostic group (neurologic $n=52$; geriatric $n=24$; psychiatric $n=14$; cancer $n=8$; and rheumatologic $n=2$ ), but medical conditions. 
Table 2 Demographic data

\begin{tabular}{lllllllll}
\hline Diagnostic groups & $\begin{array}{l}\text { Initial sample } \\
(\mathbf{n}=\mathbf{2 1 3 8 )}\end{array}$ & $\begin{array}{l}\text { Final sample } \\
(\mathbf{n}=\mathbf{2 0 9 8})\end{array}$ & $\begin{array}{l}\text { Psychiatric } \\
(\mathbf{n}=\mathbf{1 4 2})\end{array}$ & $\begin{array}{l}\text { Rheumatologic } \\
(\mathbf{n}=\mathbf{1 9 4})\end{array}$ & $\begin{array}{l}\text { Cancer } \\
(\mathbf{n}=\mathbf{1 7 8})\end{array}$ & $\begin{array}{l}\text { Medical } \\
(\mathbf{n}=\mathbf{1 1 6})\end{array}$ & $\begin{array}{l}\text { Neurologic } \\
(\mathbf{n}=\mathbf{6 5})\end{array}$ & $\begin{array}{l}\text { Geriatric } \\
(\mathbf{n}=\mathbf{1 4 0 3})\end{array}$ \\
\hline Female, $\mathrm{n}(\%)$ & $1447(67.7)$ & $1433(63.8)$ & $67(47.2)$ & $183(94.3)$ & $89(50.0)$ & $74(63.8)$ & $33(50.8)$ & $987(70.3)$ \\
Age (years), M (SD) & $73.6(15.0)$ & $73.7(14.9)$ & $* 42.0(18.0)$ & $59.2(16.9)$ & $67.4(9.7)$ & $76.1(10.3)$ & $68.8(9.8)$ & $79.8(7.9)$ \\
Range & $19-99$ & $19-99$ & $19-85$ & $21-95$ & $38-89$ & $29-92$ & $44-88$ & $55-99$ \\
\hline
\end{tabular}

SD Standard deviation

*Mean age is based on the age of $n=140$ due to missing data

\section{Psychometric properties of the rating scale}

Initial evaluation of the psychometric properties of the $0-3$ rating scale revealed $>10$ observations in each category with a frequency peak in category 3 (Table 3 ). Average category measures advanced monotonically up the rating scale, but thresholds from categories 0 to 1 , and 1 to 2 were below 1.4 logits, and threshold disordering was found between categories 1 and 2. All category outfit $M n S q$ values were $<2.0$ logits. In the final analyses, after removal of misfitting ADL items, category thresholds increased and there was no threshold disordering (Table 3).

\section{Unidimensionality}

The initial PCA of the standardized residuals revealed that $54.3 \%$ of the total variance was explained by the Rasch dimension, but the unexplained variance in the first contrast had an eigenvalue of 3.8, suggesting a second dimension with 3-4 items. Nevertheless, disattenuated correlations were above 0.7 between all clusters, supporting unidimensionality.

Still, ten items displayed both high infit and outfit (underfit) misfit: Calling for attention, Reading, Using the phone, Bowel and urine elimination volitional, Pedicuring, Writing by hand/using word processor, Driving car, Making plans for shopping, Shaving/make-up, Riding bicycle/moped. Two items only displayed high infit misfit: Manicuring, Light washing by hand, and one item displayed only high outfit misfit: Taking part in a conversation (Table 4). During the process of removing items with high infit misfit, another three items revealed high infit misfit: Going by car, Going by bus/tram/tube and Going by train/boat/aeroplane, and were therefore removed. Four items displayed overfit misfit: Washing body/bathing/showering, Dressing lower trunk, Dressing upper trunk and Undressing. These were retained as they did not pose a threat to the measurement system.

After removal of a total of 16 underfitting items, in the PCA the variance explained by the Rasch dimension increased to $58.0 \%$, whereas the eigenvalue representing variance explained by first contrast slightly decreased to 3.5 on the 31 -item measurement model. The disattenuated correlations between person measures generated based on three sets of item clusters remained above 0.7 . The DTF analysis of the variance of ability estimates across the full (47-item) version of the ADL-I and the 31 -item version is illustrated in Fig. 1 . The analysis revealed $n=49(2.3 \%)$ participants with significantly different quality of ADL task performance measures between the full version and the 31 -item version. Thus, the 16 misfitting items were not a threat to the measurement system.

\section{Person-response validity}

When evaluating person-response validity based on the 47 items in the ADL-I, $n=186$ (8.7\%) persons did not have ADL-I measures with acceptable goodness-of-fit to the Rasch model. After removal of misfitting items, the proportion of persons with misfitting ADL-I measures increased slightly to 9.1\%: psychiatric $\mathrm{n}=22(15.5 \%)$; rheumatologic $n=7$ (3.6\%); cancer $n=25$ (14.0\%); medical $\mathrm{n}=4(3.5 \%)$; neurologic $\mathrm{n}=8(12.3 \%)$ and geriatric $\mathrm{n}=125(8.9 \%)$.

\section{Differential item functioning}

Based on the 31-item version of the ADL-I, analysis of DIF based on gender revealed that the items Combing one's hair $(\mathrm{p}=0.002)$ and Weekly shopping $(\mathrm{p}<0.001)$ were relatively easier for males and Daily light cleaning $(\mathrm{p}<0.001)$ was relatively easier for females (Fig. 2).

Still, DTF analyses based on scatter plots (Figs. 3 and 4) illustrating the variance of ADL ability measures across the common and gender-specific item calibrations for males and females, respectively, showed that all person ADL ability measures fell within the 95\% confidence interval control lines, indicating no significant difference in person ADL ability measures between the common version and the gender-specific versions. Thus, indicating that the items initially displaying uniform DIF were not a threat to the measurement system.

DIF analyses based on diagnoses also revealed uniform DIF in relation to some of the items (Fig. 4). Still, patterns similar to DIF by gender were found in DTF analyses using scatter plots of the variance of ADL ability measures across the common and the diagnosis-specific item difficulty calibrations for each of the six diagnostic 


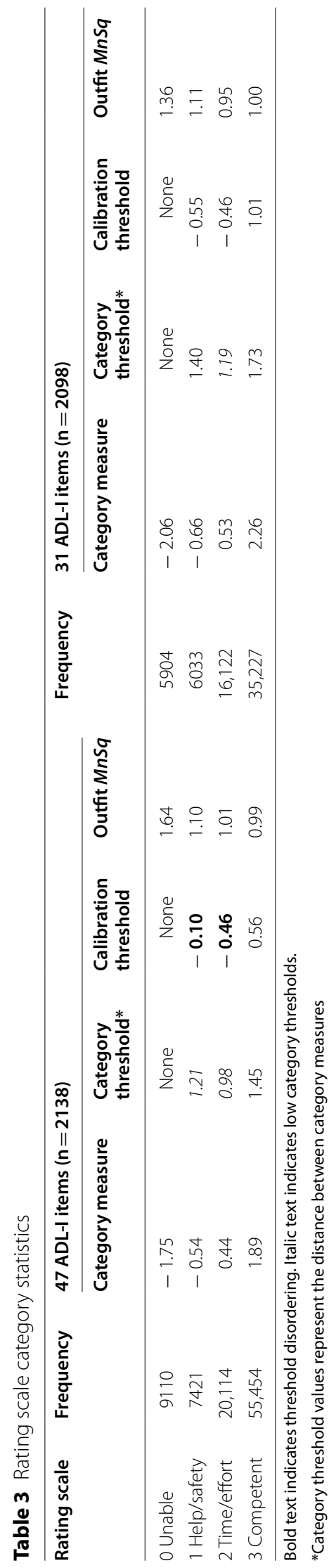


Table 4 Items measurement report

\begin{tabular}{|c|c|c|c|c|c|c|c|}
\hline \multirow[t]{2}{*}{ ADL-I item } & \multirow[t]{2}{*}{ Count } & \multirow[t]{2}{*}{ Measure } & \multirow[t]{2}{*}{$S E$} & \multicolumn{2}{|l|}{ Infit } & \multicolumn{2}{|l|}{ Outfit } \\
\hline & & & & $M n S q$ & $z$ & $M n S q$ & $z$ \\
\hline Weekly heavy cleaning (hardest item) & 2020 & 2.38 & 0.03 & 1.09 & 2.66 & 1.16 & 2.95 \\
\hline Weekly/large quantity shopping ${ }^{b}$ & 1847 & 1.80 & 0.03 & 1.04 & 1.36 & 1.04 & 0.93 \\
\hline Pedicuring & 2103 & 1.75 & 0.03 & 1.81 & 9.90 & 1.96 & 9.90 \\
\hline Riding bicyckle/moped & 687 & 1.60 & 0.04 & 1.51 & 8.71 & 1.53 & 6.76 \\
\hline Heavy washing in washing machine & 1971 & 1.57 & 0.03 & 1.12 & 4.04 & 1.08 & 2.12 \\
\hline Going by train/boat/aeroplane $\mathrm{a}^{\mathrm{a}}$ & 769 & 1.39 & 0.04 & 1.09 & 1.87 & 0.98 & -0.38 \\
\hline Light washing in washing machine & 1983 & 1.32 & 0.03 & 1.15 & 5.05 & 1.10 & 2.55 \\
\hline Driving car & 730 & 1.19 & 0.04 & 1.63 & 9.90 & 1.56 & 7.22 \\
\hline Go by bus/tram/tube ${ }^{a}$ & 1300 & 1.18 & 0.03 & 1.03 & 0.73 & 1.01 & 0.22 \\
\hline Daily/small quantity shopping & 2027 & 1.07 & 0.03 & 0.88 & -4.15 & 0.85 & -3.95 \\
\hline Daily light cleaning ${ }^{b}$ & 2050 & 1.04 & 0.03 & 0.94 & -1.95 & 1.02 & 0.40 \\
\hline Cooking a hot meal & 1874 & 0.99 & 0.03 & 1.16 & 4.86 & 1.31 & 6.96 \\
\hline Walking/moving in the neighbourhood & 2106 & 0.75 & 0.03 & 0.76 & -8.64 & 0.83 & -4.40 \\
\hline Light washing by hand ${ }^{b}$ & 1354 & 0.65 & 0.03 & 1.33 & 7.95 & 1.20 & 3.57 \\
\hline Walking/moving from one floor to another & 2100 & 0.56 & 0.03 & 0.70 & -9.90 & 0.79 & -5.14 \\
\hline Washing body/bathing/showering & 2120 & 0.46 & 0.03 & 0.58 & -9.90 & 0.66 & -8.50 \\
\hline Pulling on stockings/pantyhose/shoes & 2122 & 0.36 & 0.03 & 0.78 & -7.13 & 0.94 & -1.27 \\
\hline Washing one's hair & 2116 & 0.34 & 0.03 & 0.91 & -2.92 & 0.91 & -2.00 \\
\hline Walking/moving in and out of the house & 2119 & 0.26 & 0.03 & 0.80 & -6.51 & 0.76 & -5.26 \\
\hline Going by car ${ }^{\mathrm{a}}$ & 1884 & 0.22 & 0.03 & 1.01 & 0.29 & 1.02 & 0.30 \\
\hline Dressing lower trunk & 2129 & -0.01 & 0.03 & 0.55 & -9.90 & 0.64 & -7.71 \\
\hline Preparing a cold meal & 2060 & -0.07 & 0.03 & 0.89 & -2.85 & 0.79 & -3.98 \\
\hline Heating up liquid or prepared food & 2007 & -0.10 & 0.03 & 1.05 & 1.30 & 0.90 & -1.84 \\
\hline Transferring the body from bed to chair & 2132 & -0.15 & 0.03 & 0.72 & -7.89 & 0.86 & -2.64 \\
\hline Manicuring & 2107 & -0.15 & 0.03 & 1.38 & 8.73 & 1.27 & 4.36 \\
\hline Walking/moving from one room to another & 2130 & -0.19 & 0.03 & 0.72 & -7.99 & 0.77 & -4.40 \\
\hline Undressing & 2128 & -0.23 & 0.03 & 0.60 & -9.90 & 0.59 & -8.23 \\
\hline Dressing upper trunk & 2126 & -0.24 & 0.03 & 0.60 & -9.90 & 0.64 & -6.91 \\
\hline Transfer in bed, changing positions, turning over, sitting up & 2122 & -0.33 & 0.03 & 0.82 & -4.59 & 0.94 & -0.89 \\
\hline Making plans for shopping & 1999 & -0.48 & 0.04 & 1.57 & 9.90 & 1.54 & 6.85 \\
\hline Writing by hand/using word processor & 2105 & -0.52 & 0.04 & 1.41 & 7.98 & 1.73 & 8.91 \\
\hline Getting necessary clothes from closets/drawers & 2114 & -0.62 & 0.04 & 0.99 & -0.13 & 0.72 & -4.45 \\
\hline Getting to and from the toilet room in time & 2108 & -0.64 & 0.04 & 0.78 & -5.12 & 0.75 & -3.85 \\
\hline Arranging clothes and equipment, washing hands & 2107 & -0.76 & 0.04 & 0.70 & -6.86 & 0.55 & -7.15 \\
\hline Getting on/of toilet and cleaning one's self after elimination & 2115 & -0.77 & 0.04 & 0.78 & -4.82 & 0.68 & -4.84 \\
\hline Shaving/make-up & 1985 & -0.78 & 0.04 & 1.55 & 9.06 & 1.42 & 4.78 \\
\hline Reading & 2112 & -0.78 & 0.04 & 1.68 & 9.90 & 2.47 & 9.90 \\
\hline Getting food and liquid, cutting up food & 2129 & -0.80 & 0.04 & 1.05 & 1.06 & 0.88 & -1.64 \\
\hline Washing hands and face & 2126 & -1.10 & 0.05 & 0.84 & -3.05 & 0.63 & -4.95 \\
\hline Using the phone & 2118 & -1.27 & 0.05 & 1.47 & 6.89 & 2.07 & 8.83 \\
\hline Bowel and urine elimination volitional & 2115 & -1.29 & 0.05 & 1.37 & 5.44 & 2.04 & 8.59 \\
\hline Combing one's hair ${ }^{b}$ & 2109 & -1.29 & 0.05 & 1.14 & 2.22 & 1.10 & 1.08 \\
\hline Brushing teeth & 2121 & -1.43 & 0.05 & 1.00 & 0.04 & 1.22 & 2.11 \\
\hline Taking part in a conversation & 2121 & -1.51 & 0.06 & 1.05 & 0.73 & 1.80 & 8.33 \\
\hline Eating & 2134 & -1.66 & 0.06 & 0.98 & -0.21 & 1.34 & 2.90 \\
\hline Calling for attention & 2126 & -1.80 & 0.06 & 1.43 & 5.10 & 2.76 & 9.90 \\
\hline
\end{tabular}


Table 4 (continued)

\begin{tabular}{|c|c|c|c|c|c|c|c|}
\hline \multirow[t]{2}{*}{ ADL-I item } & \multirow[t]{2}{*}{ Count } & \multirow[t]{2}{*}{ Measure } & \multirow[t]{2}{*}{$S E$} & \multicolumn{2}{|l|}{ Infit } & \multicolumn{2}{|l|}{ Outfit } \\
\hline & & & & $\mathrm{MnSq}$ & $z$ & MnSq & $z$ \\
\hline Drinking, getting liquid from glass into mouth (easiest item) & 2132 & -1.92 & 0.07 & 1.04 & 0.52 & 1.31 & 2.41 \\
\hline Mean & 1959.6 & 0.00 & 0.04 & 1.05 & 0.00 & 1.15 & 0.60 \\
\hline SD & 362.2 & 1.07 & 0.01 & 0.32 & 6.30 & 0.50 & 5.40 \\
\hline
\end{tabular}

Bold text indicates initially misfitting ADL-I items

${ }^{a}$ Items starting to misfit, after removal of initially misfitting items

${ }^{\mathrm{b}}$ Items displaying differential item functioning (DIF) based on gender

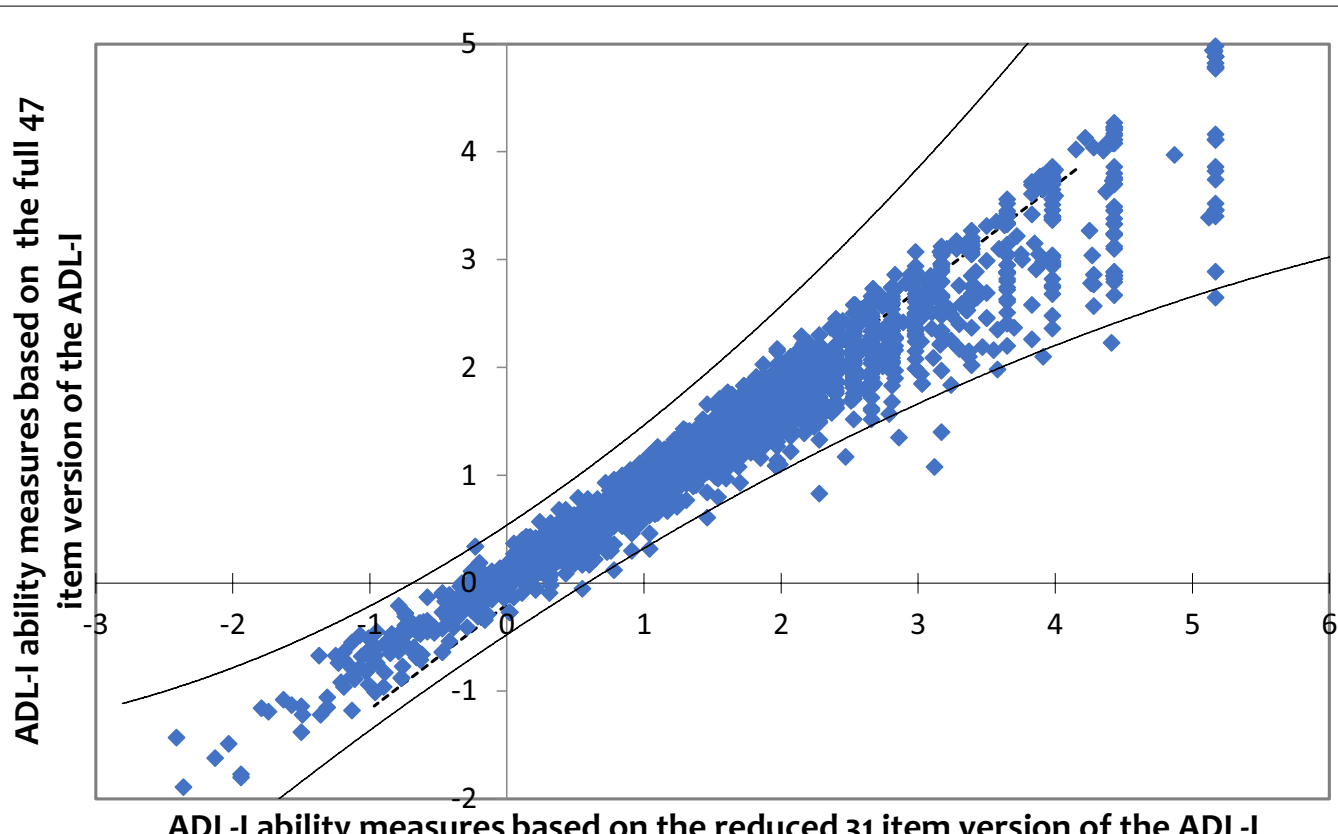

Fig. 1 Scatterplot (DTF analysis) of the variance of ability measures across the full (47-item) and reduced (31-item) version of the ADL-I. The model for invariance of measures is represented by a straight line with a slope equal to 1 (i.e. $45^{\circ}$ ) through the point representing the mean quality of ADL task performance measure from each version. Control lines, representing a 95\% confidence interval on either side of the diagonal line, drawn through the mean quality of $A D L$ task performance measures, illustrate quality of $A D L$ task performance measures displaying significant variance across the two versions [23]

subgroups. This is illustrated by an example using data on people with a neurologic condition in Fig. 5. Thus, all person ADL ability measures fell within the $95 \%$ confidence interval control lines, indicating no significant differences in person ADL ability measures between the common version and the diagnosis-specific versions of the instrument. Hence, the DIF based on diagnoses did not influence the measurement system.

\section{Reliability and precision}

The targeting of the 47-item version of the ADL-I to the participants' ADL ability (mean item difficulty measure: zero, SD 1.06; mean person ADL ability estimate: 1.51, SD 1.31) indicated that the participants had a higher mean level of ADL ability than the mean item difficulty estimate (expected to be zero logits). After removal of misfitting items and participants with maximum scores, the mean item difficulty measure SD increased to 1.22 , and the mean ADL ability measure increased to 1.59 (SD decreased to 1.28), indicating slightly diminished targeting of the 31-item version of the ADL-I. The item/person distribution map (see Fig. 6), based on the 31-item version of the ADL-I, illustrates that the items and participants were well distributed along the scale, with item and person measures well targeted to each other, indicating a small ceiling effect and no floor effect.

The initial person separation for the 47 -item version of the ADL-I was 2.75 (reliability $=0.88$ ) indicating that 

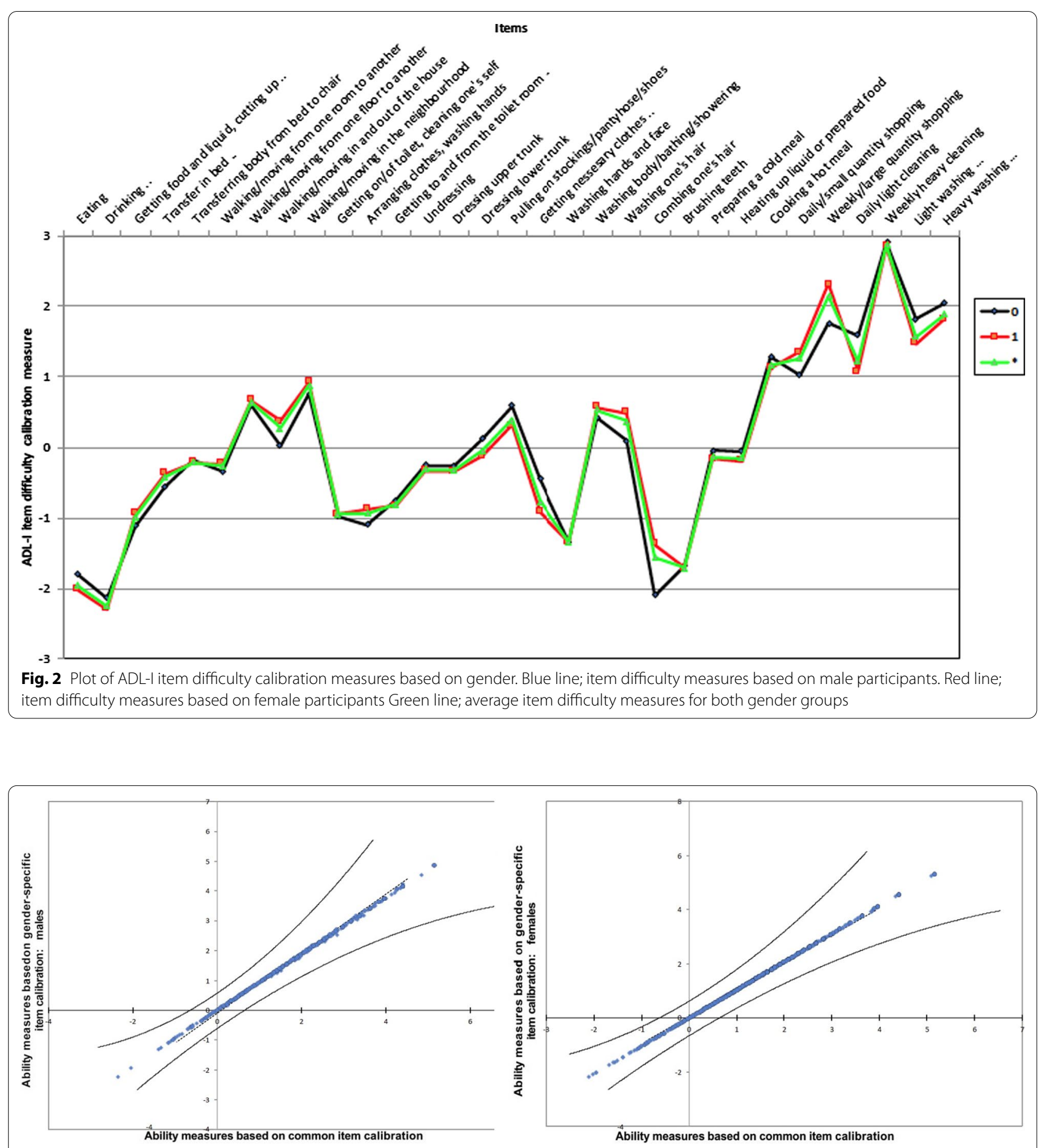

Fig. 3 Scatter plot of ADL-I ability measures based on common item calibration and ADL-I ability measures based on gender-specific item calibrations

the items separated the persons into three significantly different levels of ADL ability [42]. After removal of misfitting items the person separation index improved (2.99; reliability $=0.90$ ).

\section{Discussions}

As the number of persons diagnosed with multi-morbidity is increasing, there is a need for generic instruments to be able to assess, measure and compare ADL 


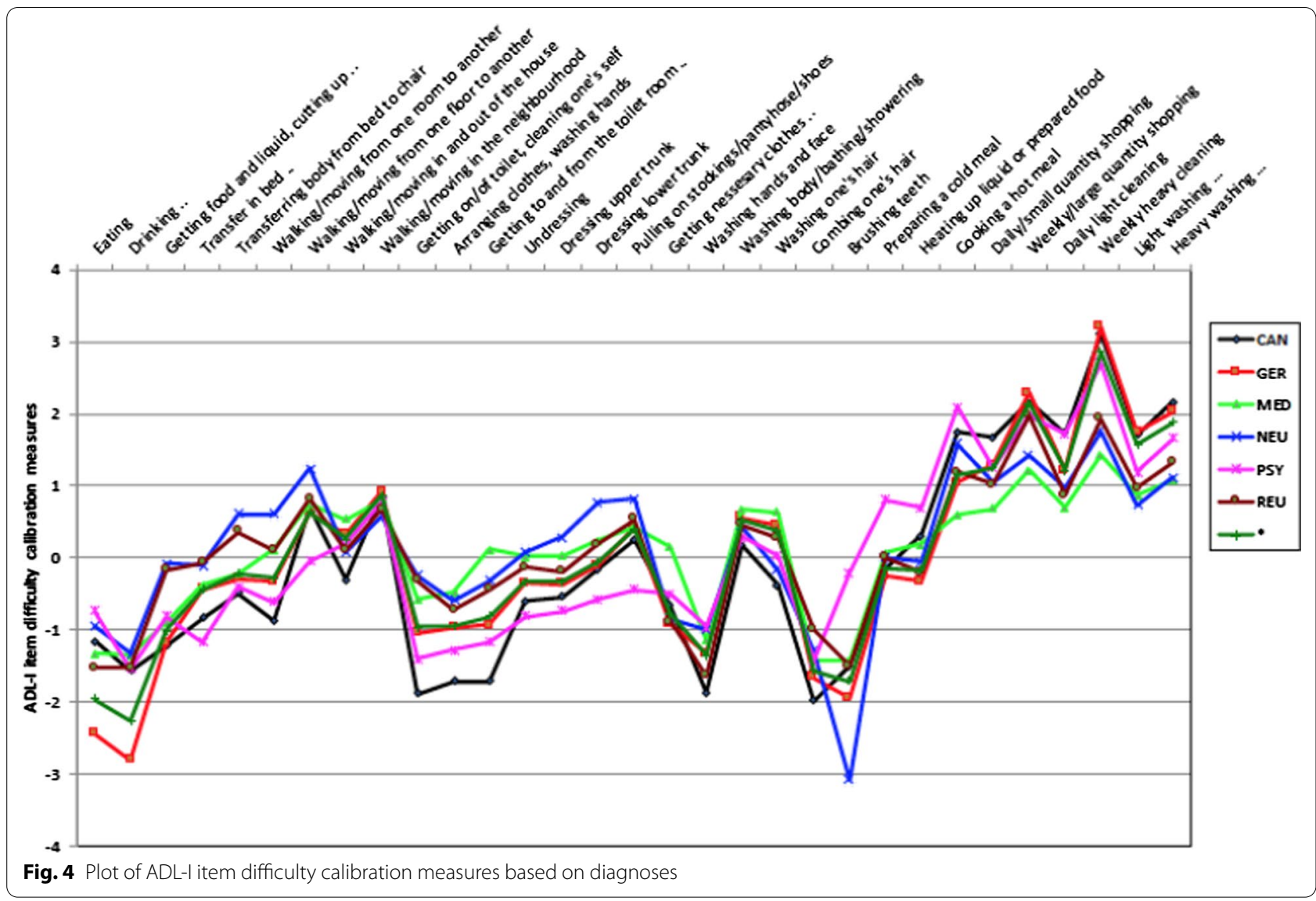

ability across gender and diagnoses. Since the ADL-I was developed as a generic instrument to be used across gender and diagnostic groups in rehabilitation research and clinical practice, the aim of this study was to examine the psychometric properties of the ADL-I applied among males and females living with various chronic conditions. While the ADL items displaying misfit overall did not represent a threat to the measurement system, removal of the misfitting items improved rating scale functioning and increased the sensitivity of the ADL-I ability measures. When the misfitting ADL items were removed, the results overall suggested that the ADL-I is producing valid and reliable measures across gender and diagnostic groups among persons within a broad range of ADL ability, providing evidence to support generic use of the ADL-I.

There might be several explanations to the misfitting items revealed in the present study. The majority of items misfitting belonged to two domains: communication and transportation. Items related to communication have been found to misfit in other ADL instruments [16, 44, 45]. In a study involving the ADL-focused Occupationbased Neurobehavioural Evaluation (A-ONE), two communication items: Comprehension and Expression, were found to misfit [44], and consequently removed from the measurement system in a recent study on the Japanese version of the A-ONE [46]. Similarly, in a Rasch analysis of the Functional Independence Measure $\left(\mathrm{FIM}^{\mathrm{TM}}\right)$, similar communication items: Comprehension and Expression, were reported to misfit [45]. Also, in a Rasch analysis of the ADL-Observation (ADL-O), an instrument similar to the ADL-I, but based on observation, the same five communication items were displaying misfit [16]. Like in the present study, DTF analysis of the ADL-O suggested that the misfitting communication items did not disturb the measurement system, supporting retaining the items in the instrument. Still, the fact that communication items seem to misfit across a range of ADL instruments, suggest that communication represents a dimension not belonging to the concept of ADL, supporting permanent removal of the five ADL-I communication items from the ADL-I.

Items related to transportation have also been found to misfit in other instruments $[16,45]$. In the Rasch analysis of the ADL-O, the item of Driving did display misfit [16] and in the Instrumental Activity Measure (IAM) the item Public Transport was found to misfit [45]. Again, as the DTF analysis suggested that the misfitting transportation 


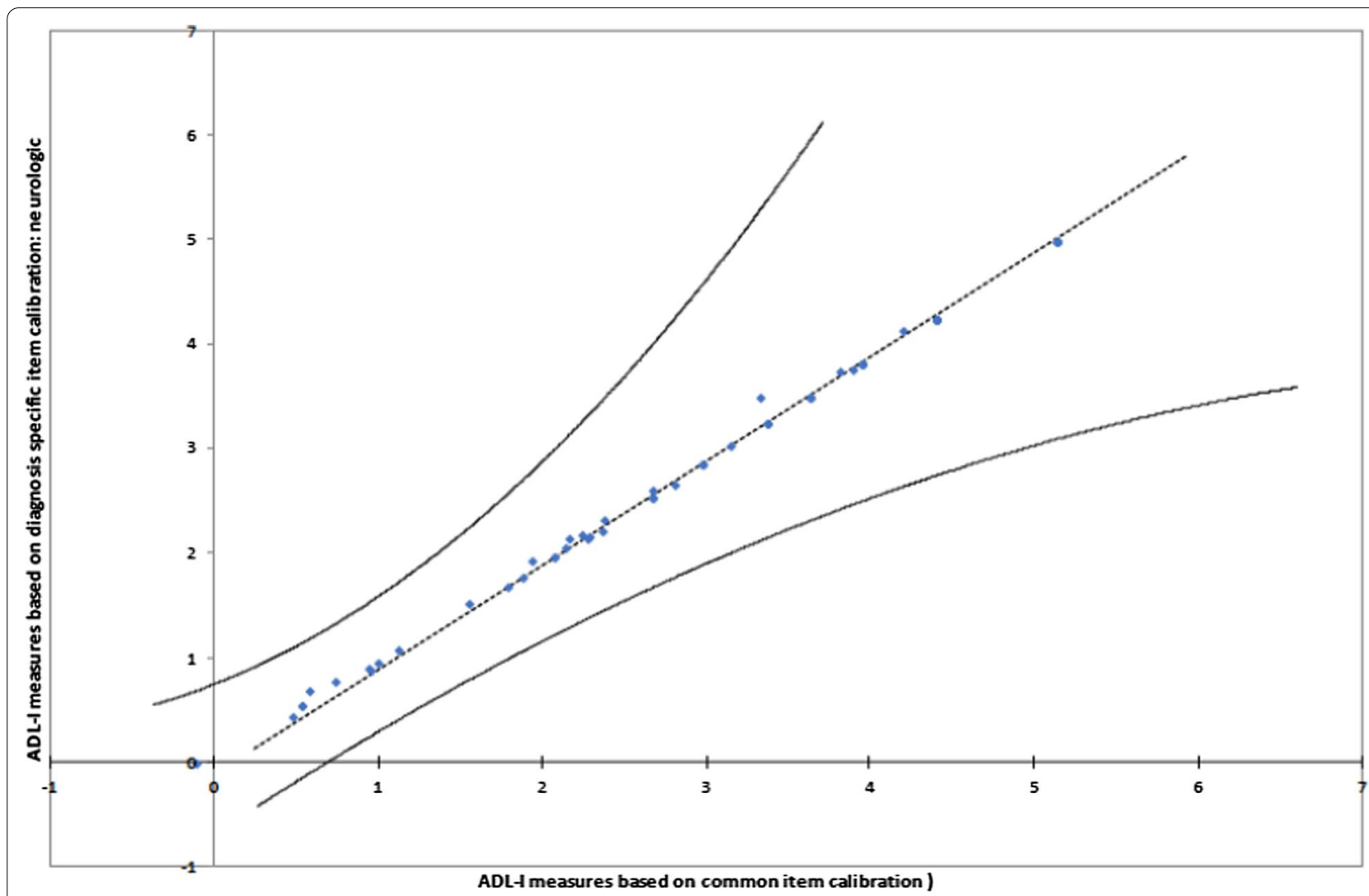

Fig. 5 Scatter plot of ADL-I ability measures based on the common item calibration and ADL-I ability measures based on a diagnosis-specific item calibration (neurologic)

(See figure on next page.)

Fig. 6 Item-person distribution map-31 item version of the ADL-I. The most difficult items and the most able participants at the top. Each '\#' is 10 persons, each ". is 1 to 9. Note: Each item is shown in the three columns representing different rating scale measures. Items: Center $=$ the mean item difficulty calibrations; Items: Bottom = measure level corresponding to a probability of 0.5 of being rated in (or exceeding) the lowest category of the rating scale; Item: Top = measure level corresponding to a probability of 0.5 of being rated in (or falling below) the highest category of the rating scale

items did not influence the measurement system, it can be discussed whether the five transportation items in the ADL-I should be removed or retained.

It is well established that items concerning bowl and bladder functioning often display misfit in traditional ADL scales like the Barthel Index $[47,48]$ and FIM $^{\mathrm{TM}}[45$, $49,50]$. One explanation could be that bowl and bladder functioning conceptually is addressed as body functioning (continence) rather than the ability to handle actions related to bowel and urine elimination. Hence, the question posed may be focused on whether the person is continent or not, rather than the ability to e.g., schedule frequent toilet visits or handle aids such as incontinence pads or urinals, if incontinent. Since the ability to handle actions related to bowel and urine elimination, being incontinent or not, is important for independent living, the item of bowel and urine elimination in the ADL-I should be contained but reformulated to more clearly reflect the intended content.

The remaining five items displaying misfit involve Shaving/make-up, Manicuring, Pedicuring, Making plans for shopping, and Light washing by hand, all of which seem relevant in an ADL instrument. The items in the ADL-I was originally adopted from the ADL Taxonomy-a classification of ADL tasks in twelve ADL domains, carefully developed in several steps $[19,20]$. Still, based on the present results, some of these items may need clarification and reformulation. For example, the item of Shaving/make-up covers two separate ADL tasks in one and, along with the items Making plans for shopping, and Light 


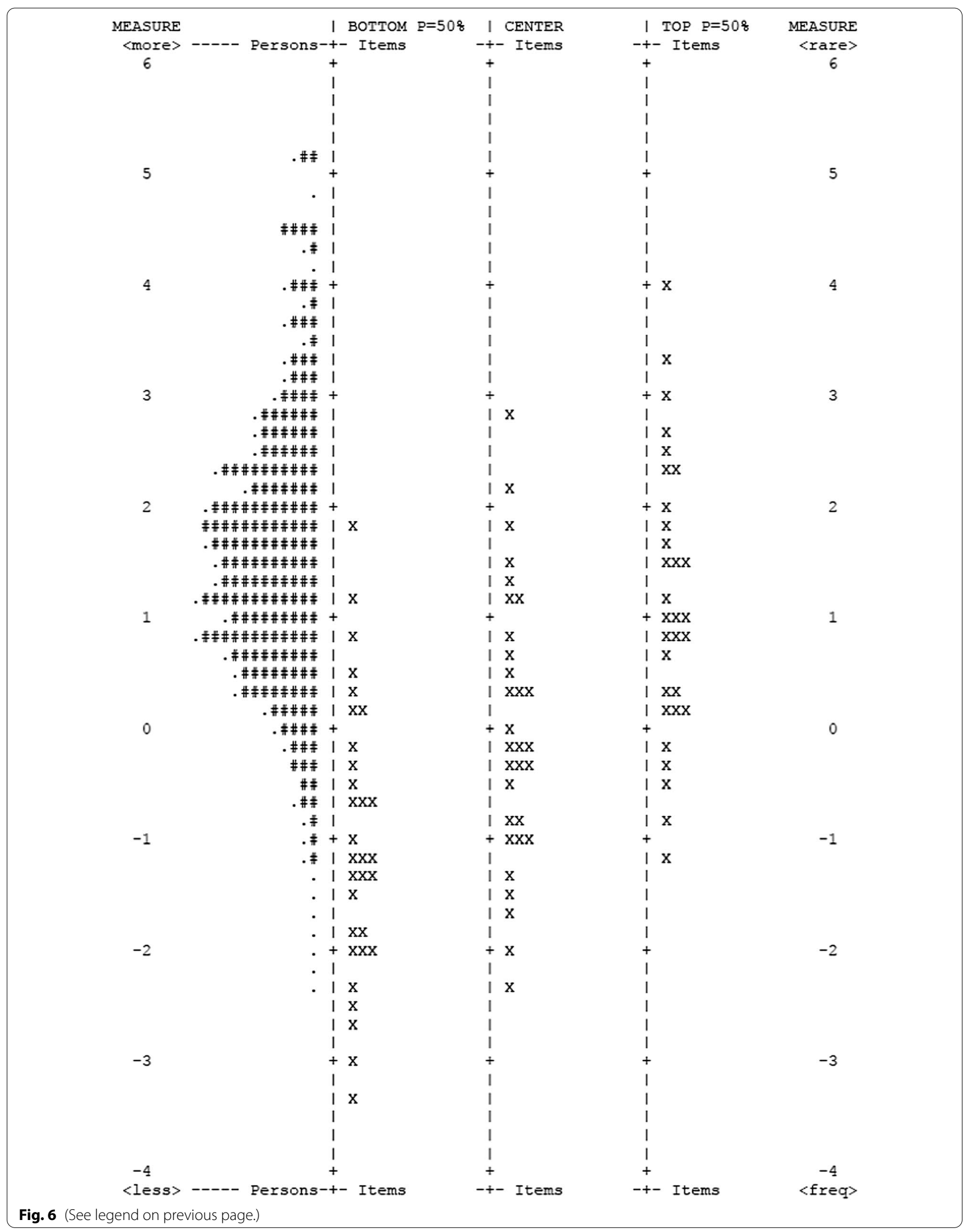


washing by hand, it represents items not all people do. In terms of the two items concerning nail care, one reason for misfit could be that answers were based on what the person did (regularly visiting e.g., a podiatrist) rather than what he or she was able to perform himself/herself. Since ADL-I is designed to assess the ability to perform ADL tasks in a safe, efficient, effortless and independent manner, it is necessary that the interviewers ask what the person is able to, not what he or she actually does.

The analysis for DIF by gender identified two items (Combing one's hair and Weekly shopping) relatively easier for males and one item (Daily light cleaning) relatively easier for females. Similar examples of DIF have previously been observed in a study by Fleishman et al. where elderly females were more likely to receive help with shopping than elderly males, whereas elderly males potentially needed more help doing light housework [51]. It has been suggested that these examples of DIF by gender in part can been attributed to historical gender roles $[52,53]$, explaining how males and females living together typically take charge of different IADL tasks based on roles, habits, routines and preferences. Still, the difficulty of a task may not just be explained by level of routine, but also variations within the task across gender. One example may be that most males prefer a low-maintenance hairstyle, whereas many females wear their hair in a way requirering styling.

Likewise, DIF by diagnoses were identified across the ADL-I items, indicating that item difficulty estimates were sensitive to the characteristics of the diagnostic groups. Still, the DIF based on diagnoses did not influence the measurement system as no significant differences in person ADL ability measures were identified between the common version and the diagnosis-specific versions of the ADL-I. Similar findings have been reported in a study involving the FIM $^{\mathrm{TM}}$ employed in two diagnostic groups; stroke and orthopedic impairments [54]. While several items were displaying DIF by diagnoses e.g., eating and bowel continence, minimal influence on FIM measures was identified.

While the ADL-I items displaying misfit or DIF by gender or diagnoses did not influence the overall measurement system, removal of misfitting ADL items improved the rating scale in terms of increased category thresholds and no threshold disordering. Moreover, after removal of almost $1 / 3$ of the initial items, the instrument became more sensitive to detect differences, as indicated by the increased person separation index. One reason could be that most items removed represented other dimensions than ADL e.g., body functions (bowl/bladder), communication and transportation. Still, a few misfitting items do represent the ADL dimension. Thus, other reasons for misfit e.g., unclear definitions of the items should be explored and if possible, resolved. Also, since up to 5\% of items on a scale are expected to misfit by chance [55], 1-2 items demonstrating misfit may be kept in the scale.

Since the 47 ADL items of the ADL-I, based on the ADL Taxonomy, previously have been reported as relevant for clinical use, all ADL items can be retained in the ADL-I for the purposes of assessing and describing single client's quality of ADL task performance, and identifying targets in rehabilitation processes. For measuring self-reported quality of ADL task performance in clinical and research only items displaying fit to the Rasch measurement model should be used. For this purpose, conversion tables can be made available based on the 31-item version. Future studies are needed to evaluate the clinical utility of the ADL-I, including the ease to use in clinical research and practice [56].

\section{Strength and limitations}

The inclusion of a large and diverse study sample representing variation across age, gender, diagnoses and selfreported quality of ADL task performance was a great strength of the Rasch analysis. Moreover, the data was extracted from a research database containing ADL-I data from a range of research studies. Hence, all data were collected for research purposes by trained occupational therapists, supporting data quality. As the database is constructed based on anonymized datasets only including age, gender, diagnosis and raw ADL-I ratings, the study was limited in terms of describing the study sample in more detail. Moreover, the sample sizes for persons with medical, rheumatological, oncological, neurological, and psychiatric chronic conditions may be considered small and potentially result in unstable measures. Still, according to Linacre, a sample size of 64 to 144 persons will provide stable item calibrations and person ability measures within 0.5 logits (CI 95\%) [57].

\section{Conclusions}

The 31-item version of the ADL-I is producing valid and reliable measures across gender and diagnostic groups among persons within a broad range of ADL ability.

\footnotetext{
Abbreviations

ADL: Activities of daily living; ADL-I: ADL interview; ADL-O: ADL observation; AMPS: Assessment of motor and process skills; A-One: ADL-focused occupation-based neurobehavioural evaluation; COPD: Chronic obstructive pulmonary disease; DIF: Differential item functioning; DTF: Differential test functioning; FIM ${ }^{\mathrm{TM}}$ : Functional independence measure; IADL: Instrumental activities of daily living; MnSq: Mean square; PADL: Personal activities of daily living; PCA: Principal component analysis; PCM: Partial credit model; RSM: Rasch Rating Scale model.
} 


\section{Acknowledgements}

The authors would like to thank the participants and the occupational therapists who conducted the ADL-I interviews. Further, database consultant Christian Cato is gratefully acknowledged for database support.

\section{Authors' contributions}

Study design: EW, KTN. Data extraction: KTN, EW. Analyses: EW. Writing first draft: EW, KTN. Critical revision of manuscript: EW, AK, KTN. All authors read and approved the final manuscript.

\section{Funding}

This work was supported by the Danish Association of Occupational Therapists [PP2 - R70- A1509] and The Oak Foundation [OCAY-18-774-OFIL].

\section{Availability of data and materials}

The datasets used and/or analysed during the current study are available from the corresponding author on reasonable request.

\section{Declarations}

\section{Ethics approval and consent to participate}

The local health research committee was contacted prior to initiating the study and deemed the study exempt from approval. The database is established in accordance with Danish legislation and data is anonymized according to the General Data Protection Regulation [58].

\section{Consent for publication}

Not applicable.

\section{Competing interests}

The authors declare that they have no competing interests.

\section{Author details}

${ }^{1}$ The ADL Unit, The Parker Institute, Copenhagen University Hospital Bispebjerg-Frederiksberg, Nordre Fasanvej 57, 2000 Frederiksberg, Denmark. ${ }^{2}$ Occupational Science and Occupational Therapy, User Perspectives and Community-Based Research, Department of Public Health, University of Southern Denmark, J. B. Winsløwsvej 9a, 5000 Odense, Denmark. ${ }^{3}$ Faculty of Health and Society, Malmö University, 20506 Malmö, Sweden. ${ }^{4}$ Department of Occupational Therapy, University College of Northern Denmark, Selma Lagerløfsvej 2, 9220 Aalborg, Denmark.

Received: 7 July 2021 Accepted: 11 October 2021

Published online: 18 October 2021

\section{References}

1. Avlund K, Schultz-Larsen K, Kreiner S. The measurement of instrumental ADL: content validity and construct validity. Aging (Milano) 1993:5:371-83.

2. Wade D. Rehabilitation - a new approach. Part four: a new paradigm, and its implications. Clin Rehabil. 2016;30(2):109-18.

3. Mahoney F, Barthel D. Functional evaluation: the Barthel Index. Md State Med J. 1965;14:61-5.

4. Kidd D, Stewart G, Baldry J, Johnson J, Rossiter D, Petruckevitch A, et al. The functional Independence measure: a comparative validity and reliability study. Disabil Rehabil. 1995;17(1):10-4.

5. Fisher A, Jones K. Assessment of Motor and Process Skills: development, standardization, and administration manual. Seventh edition, revised ed. Fort Collins: Three Star Press, Inc.; 2012.

6. Fisher A, Jones K. Assessment of motor and process skills: user manual. 8th ed. Fort Collins: Three Star Press Inc.; 2014.

7. Fisher AG, Jones KB. Assessment of motor and process skills: development, standardization, and administration manual. 7th ed. Fort Collins: Three Star Press, Inc.; 2012

8. Wæhrens E, Bliddal H, Danneskiold-Samsøe B, Lund H, Fisher AG. Differences between questionnaire-and interview-based measures of activities of daily living (ADL) ability and their association with observed
ADL ability in women with rheumatoid arthritis, knee osteoarthritis, and fibromyalgia. Scand J Rheumatol. 2012;41(2):95-102.

9. Nielsen KT, Wæhrens EE. Occupational therapy evaluation: use of selfreport and/or observation? Scand J Occup Ther. 2015;22(1):13-23.

10. Wæhrens E, Brandt $\AA$, Peoples H, la Cour K. Everyday activities when living at home with advanced cancer: a cross-sectional study. Eur J Cancer Care. 2020:29:e13258.

11. Wæhrens E. Measuring quality of occupational performance based on self-report and observation. Development and validation of instruments to evaluate ADL task performance, Umeå University, Sweden; 2010.

12. Bendixen $H$, Wæhrens $E$, Wilcke J, Sørensen L. Self-reported quality of ADL task performance among patients with COPD exacerbations. Scand J Occup Ther. 2014;21(4):313-20.

13. Lindahl-Jacobsen L, Hansen D, Wæhrens E, la Cour K, Søndergaard J. Performance of activities of daily living among hospitalized cancer patients. Scand J Occup Ther. 2015;22(2):137-46.

14. Nielsen K, Klokker L, Guidetti S, Wæhrens E. Self-reported quality of activities of daily living (ADL) task performance in persons with chronic conditions. Int J Ther Rehabil. 2021;28(4):1-10.

15. Winkel $\mathrm{A}$, Langberg $\mathrm{H}$, Wæhrens $\mathrm{E}$. Reablement in a community setting. Disabil Rehabil. 2014;37(15):1347-52.

16. Wæhrens $E$, Fisher A. Developing linear ADL ability measures based on the ADL taxonomy: a Rasch analysis. Scand J Occup Ther. 2009;16:159-71.

17. Goodman R, Posner S, Huang E, Parekh A, Koh H. Defining and measuring chronic conditions: imperatives for research, policy, program, and practice. Prev Chronic Dis. 2013;10:E66.

18. Wæhrens E, Nielsen K. The ADL-Interview (ADL-I) manual. Copenhagen; 2020

19. Sonn U, Törnquist $K$, Svensson E. The ADL taxonomy-from individual categorical data to ordinal categorical data. Scand J Occup Ther. 1999:6:1120.

20. Törnquist K, Sonn U. Towards an ADL taxonomy for occupational therapists. Scand J Occup Ther. 1994;1:6976.

21. Linacre J. Winsteps ${ }^{\circledR}$ Rasch measurement computer program. Beaverton: Winsteps.com; 2020.

22. Wright B. Logits? Rasch Meas Trans. 1993;993(7):288.

23. Bond TG, Fox CM. Applying the Rasch model. Fundamental measurement in the human science. 3rd ed. Mahwah: Lawrence Erlbaum Associates; 2015 .

24. Wright B, Masters G. Rating scale analysis. Rasch measurement. Chicago: MESA Press; 1982

25. Linacre J. Winsteps ${ }^{\circledR}$ Rasch measurement computer program User's Guide. Beaverton: Winsteps.com; 2020.

26. Masters G. A rasch model for partial credit scoring. Psychometrika. 1982:47:149-74.

27. Andrich D. A rating formulation for ordered response categories. Psychometrika. 1978:43:561-73.

28. Linacre J, Wright B. Construction of measures from manyfacet data. J Appl Meas. 2002:3:486-512.

29. Linacre J. Investigating rating scale category utility. J Outcome Meas. 1999:3:103-22.

30. Linacre J. Category disordering vs. step (threshold) disordering. Rasch Meas Trans. 1999:13:675.

31. Linacre J. Differential item and test functioning (DIF \& DTF). Rasch Meas Trans. 2002:16:889.

32. Smith EV. Detecting and evaluating the impact of multidimensionality using item fit statistics and principal component analysis of residuals. J Appl Meas. 2002;3:20531.

33. Linacre JM. A Users guide to winsteps ministep Rasch-model computer programs. Program Manual 4.7.0. Available at: http://www.winsteps. com/a/Winsteps-Manual.pdf: Winsteps.com; 2020.

34. Smith $A B$, Rush $R$, Fallowfield $\sqcup$, Velikova G, Sharpe M. Rasch fit statistics and sample size considerations for polytomous data. BMC Med Res Methodol. 2008:8(33):1-11.

35. Nilsson I, Fisher AG. Evaluating leisure activities in the oldest old. Scand J Occup Ther. 2006;13:31-7.

36. Patomella AH, Tham K, Kottorp A. P-drive: assessment of driving performance after stroke. J Rehabil Med. 2006:38:273-9.

37. Kottorp A, Bernspang B, Fisher AG. Validity of a performance assessment of activities of daily living for people with developmental disabilities. J Intellect Disabil Res. 2003:47:597-605. 
38. Draba R. The identification and interpretation of item bias. Research Memorandum No 25. Available at: http://www.rasch.org/memo25.htm 1977.

39. Mantel N. Chi-square tests with one degree of freedom; extensions of the Mantel-Haenszel procedure. Am Stat Assoc. 1963;58:690-700.

40. Mantel N, Haenszel W. Statistical aspects of the analysis of data from retrospective studies of disease. J Natl Cancer Inst. 1959;22:719-48.

41. Kottorp A, Malinowskya C, Larsson-Lund M, Nygård L. Gender and diagnostic impact on everyday technology use: a differential itemfunctioning (DIF) analysis of the Everyday Technology Use Questionnaire (ETUQ). Disabil Rehabil. 2019;41(22):2688-94.

42. Fisher WP. Reliability, separation, strata statistics. Rasch Meas Trans. 1992:6(3):238.

43. Wright B. Reliability and separation. Rasch Meas Trans. 2008;9:472.

44. Arnadottir G, Fisher AG. Rasch analysis of the ADL Scale of the A-ONE. Am J Occup Ther. 2007;62:51-60.

45. Andrén E, Grimby G. Activity limitations in personal, domestic and vocational tasks: a study of adults with inborn and early acquired mobility disorders. Disabil Rehabil. 2004;26(5):262-71.

46. Higashi Y, Takabatake S, Matsubara A, Nishikawa K, Shigeta H, Arnadottir G. Reliability and validity of the Japanese version of the ADL-focused Occupation-based Neurobehavioural Evaluation (A-ONE J): applying Rasch analysis methods. Hong Kong J Occup Ther. 2019;32:32-40.

47. van Hartingsveld F, Lucas C, Kwakkel G, Lindeboom R. Improved interpretation of stroke trial results using empirical Barthel item weights. Stroke. 2006;37(1):162-6.

48. Küçükdeveci A, Yavuzer G, Tennant A, Süldür N, Sonel B, Arasil T. Adaptation of the modified Barthel Index for use in physical medicine and rehabilitation in Turkey. Scand J Rehabil Med. 2000;32(2):87-92.

49. Christopher R, Pretz C, Kean J, Heinemann A, Kozlowski A, Bode R, et al. A Multidimensional Rasch analysis of the functional independence measure based on the national institute on disability, independent living, and rehabilitation research traumatic brain injury model systems national database. J Neurotrauma. 2016;33(14):1358.
50. Küçükdeveci A, Yavuzer G, Elhan A, Sonel B, Tennant A. Adaptation of the functional independence measure for use in Turkey. Clin Rehabil. 2001;15(3):311-9.

51. Fleishman J, Spector W, Altman B. Impact of differential item functioning on age and gender differences in functional disability. J Gerontol B Psychol Sci Soc Sci. 2002;57B(5):S275-84.

52. Treas J, Lui J. Studying housework across nations. J Fam Theor Rev. 2013;5:135-49.

53. Lutomskia J, Krabbec $P$, den Elzend W, Olde-Rikkerta M, Steyerberge E, Muntingaf $M$, et al. Rasch analysis reveals comparative analyses of activities of daily living/instrumental activities of daily living summary scores from different residential settings is inappropriate. J Clin Epidemiol. 2016;74:207-17.

54. Li C, Romero S, Bonilha H, Simpson K, Simpson A, Hong l, et al. Linking existing instruments to develop an activity of daily living item bank. Eval Health Prof. 2018;41(1):25-43.

55. Smith R. The distributional properties of Rasch item fit statistics. Educ Psychol Meas. 1991;51:541-65.

56. Carrozzino D, Patierno C, Guidi J, Berrocal Montiel C, Cao J, Charlson $\mathrm{M}$, et al. Clinimetric criteria for patient-reported outcome measures. Psychother Psychosom. 2021;90:222-32.

57. Linacre JM. Sample size and item calibration stability. Rasch Meas Trans. $1994 ; 7: 328$.

58. EU General Data Protection Regulation (GDPR): Regulation (EU) 2016/679 of the European Parliament and of the Council of 27 April 2016 on the protection of natural persons with regard to the processing of personal data and on the free movement of such data, and repealing Directive 95/46/EC (General Data Protection Regulation) OJ 2016 L 119/1 (2016).

\section{Publisher's Note}

Springer Nature remains neutral with regard to jurisdictional claims in published maps and institutional affiliations.
Ready to submit your research? Choose BMC and benefit from:

- fast, convenient online submission

- thorough peer review by experienced researchers in your field

- rapid publication on acceptance

- support for research data, including large and complex data types

- gold Open Access which fosters wider collaboration and increased citations

- maximum visibility for your research: over $100 \mathrm{M}$ website views per year

At BMC, research is always in progress.

Learn more biomedcentral.com/submissions 